Case Report:

\title{
Multifocal Intradural Extramedullary Anaplastic Ependymoma With Intracranial Involvement at Presentation: A Case Report
}

\author{
Khashayar Mozaffari ${ }^{1^{*+}}$ Q, Michael A. Stellon ${ }^{1+}$, Eric J. Chalif ${ }^{1}$, ${\text { Michael K. } \text { Rosner }^{1}}^{\mathrm{C}}$
}

1. Department of Neurological Surgery, George Washington University Hospital, Washington, DC, USA

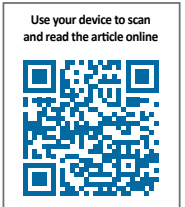

Citation Mozaffari Kh, Stellon MA, Chalif EJ, Rosner MK. Multifocal Intradural Extramedullary Anaplastic Ependymoma With Intracranial Involvement at Presentation: A Case Report. Iran J Neurosurg. 2021; 7(3):159-164. http://dx.doi.org/10.32598/ irjns.7.3.7

: http://dx.doi.org/10.32598/irjns.7.3.7

(ᄄ) 08

Article info:

Received: 08 May 2021

Accepted: 22 Jun 2021

Available Online: $01 \mathrm{Jul} 2021$

Keywords:

Ependymoma, Drop

metastasis, Surgical resection,

Radiation therapy

\section{ABSTRACT}

Background and Importance: Ependymomas are a rare malignant neoplasm. Multifocal intradural extramedullary anaplastic ependymomas are even more of a rare entity with much of the current knowledge derived from case reports. We presented a case of a multifocal intradural extramedullary anaplastic ependymoma with intracranial involvement at presentation.

Case Presentation: A 53-year-old male presented with urinary symptoms. Magnetic resonance imaging revealed two lesions along the spinal cord and two lesions, intracranially. Histopathological examination was consistent with the World Health Organization grade III anaplastic ependymoma. The patient was treated with the gross total resections of spinal cord lesions, followed by radiation therapy to the resection cavities and intracranial lesions. At the 10-month follow-up visit, he reported almost complete resolution of symptoms, and magnetic resonance imaging revealed no recurrence.

Conclusion: Despite their rarity, ependymomas should be considered as the differentia diagnosis when evaluating spinal tumors. Gross total resection followed by targeted radiotherapy appears to be an effective treatment modality for high-grade lesions.

\section{* Corresponding Author:}

Khashayar Mozaffari, BS.

Address: Department of Neurological Surgery, George Washington University Hospital, Washington, DC, USA

Tel: +949 (226) 9784

E-mail: kmozaffari2013@gmail.com

+ Joint First Authorship Statement:

Khashayar Mozaffari and Michael Shelton contributed equally to this paper and have shared the article's first authorship. 


\section{Highlights}

- Ependymomas can present as multifocal tumors.

- Intradural extramedullary ependymomas are very rare.

- Surgery and adjuvant radiation are a good treatment for high-grade ependymomas.

\section{Plain Language Summary}

Ependymomas are rare tumors that originate from the central nervous system. Given their rarity, there are no clearly defined guidelines about their management, particularly in cases where tumors present in multiple regions of the brain and spinal cord. In this report, the authors present an interesting case of a 53-year-old male who presented to the emergency department with difficulty urinating and a sensation of incomplete bladder emptying. Imaging studies revealed the presence of tumors in the brain and spinal cord consistent with the diagnosis of ependymoma. The patient received surgery along with radiation therapy and has been in remission since. We hope the findings of our case report add valuable information to the field of medicine by raising awareness about these tumors and encouraging physicians to consider ependymomas on their differential diagnoses when evaluating patients. Additionally, given our success with treatment, we hope to contribute to the existing literature on the treatment of these tumors, and the development of management guidelines

\section{Background and Importance}

pendymomas are a rare malignant neoplasm with an incidence of $0.6 /$ million $[1,2]$. The average age of diagnosis in adults is $35-45$ years, with a slight male predilection [1, 3, 4]. The World Health Organization (WHO) further classifies ependymomas as grades I-III based on predicted clinical behavior [5]. The 5-year survival rate of ependymal tumors is reported as $84.5 \%[5,6]$. Spinal ependymomas tend to present similarly to space occupying the lesions of the spinal cord. Moreover, the most common presenting symptoms consist of pain, paresthesia, weakness, and gait disturbances $[1,3,4,7]$. Diagnosis is based on clinical presentation, radiographic evaluation, and biopsy [8].

For the management of ependymomas, the current literature suggests performing Gross Total Resection (GTR), as it is associated with better therapeutic outcomes [9]. If GTR is impossible, or if the tumor is the WHO grade III, most large case series recommend using adjuvant radiotherapy $[10,11]$; although there is a lack of consensus in the respect.

Given the low incidence of ependymomas [2], multifocal Intradural Extramedullary (IDEM) anaplastic ependymomas are even more of a rare entity with much of the current knowledge derived from case reports [12-14]. We presented a case of a multifocal IDEM WHO grade III anaplastic ependymoma with intracranial involvement at presentation. The patient consented to the possibility of his data being used for research. Besides, the study was approved by the Institutional Review Board (IRB). All protected health information was secured according to the Health Insurance Portability and Accountability Act (HIPAA).

\section{Case Presentation}

A 53-year-old male with a remote history of resected basal cell carcinoma of the chest presented to the emergency department. He complained of one and a half months of difficulty initiating micturition and a sensation of incomplete bladder emptying. Cystoscopy, at that time, demonstrated inflammation concerning for a urinary tract infection. The reported patient was treated with antibiotics and noted only slight symptomatic improvement. Two weeks later, he returned to his primary care provider and reported continued urinary difficulties along with paresthesia in his abdomen, back, and bilateral lower extremities. His urinary symptoms progressed to the point of requiring self-catheterization. He also reported mild sexual dysfunction but denied issues with defecation. Initial neurologic examination was notable for a band of reported abnormal sensation at the T6 dermatomal level which extended throughout bilateral lower extremities. His strength was $5 / 5$ throughout, without pronator drift or pathological reflexes. 


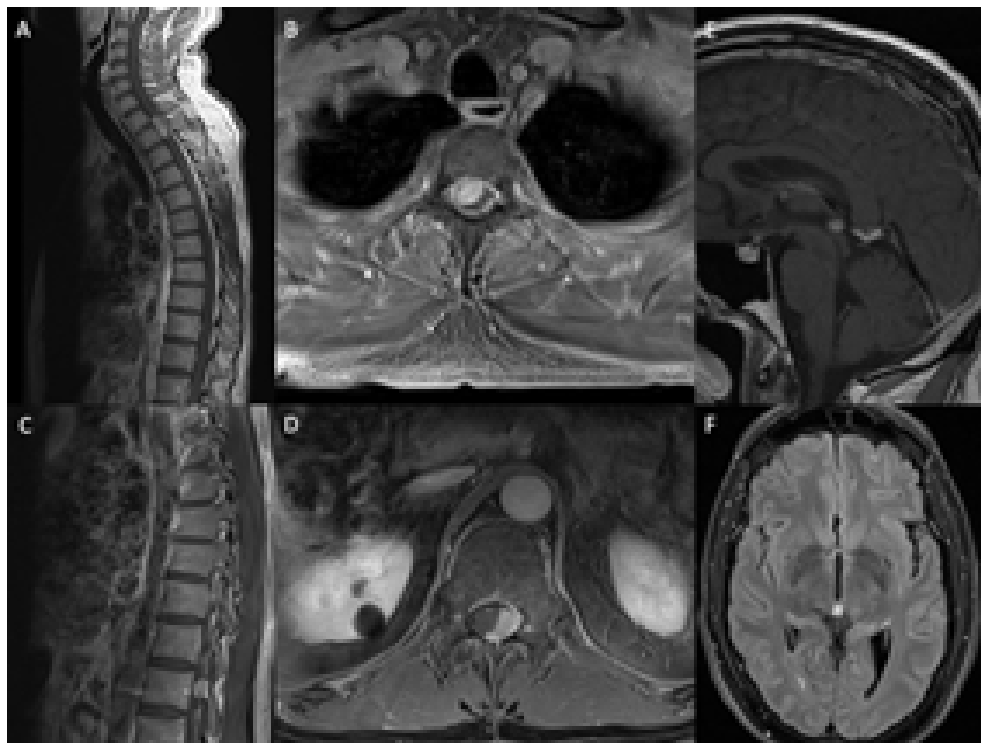

Figure 1. Images of the spine

A) Sagittal T1-weighted post-contrast image of the spine demonstrating a homogeneously enhancing intradural extramedullary lesion at T1-2 measuring $2.6 \mathrm{~cm}$ in the craniocaudal plane;

B) Axial T1-weighted post-contrast image of the spine demonstrating a homogeneously enhancing intradural extramedullary lesion at T1-2 measuring $1.0 \mathrm{~cm} \times 1.3 \mathrm{~cm}$ displacing the spinal cord laterally;

C) Sagittal T1-weighted post-contrast image of the spine demonstrating a homogeneously enhancing intradural extramedullary lesion at T12 measuring $1.6 \mathrm{~cm}$ in the craniocaudal plane;

D) Axial T1-weighted post-contrast image of the spine demonstrating a homogeneously enhancing intradural extramedullary lesion at T12 measuring $1.4 \mathrm{~cm} \times 0.8 \mathrm{~cm}$ displacing the spinal cord laterally;

E) Sagittal T1-weighted post-contrast image demonstrating two intracranial lesions, a $4 \mathrm{~mm}$ focus of enhancement along the right aspect of the pituitary stalk insertion in the superior sella, and a $9 \mathrm{~mm}$ focus enhancement in the pineal gland;

F) Axial Flair image demonstrating a $9 \mathrm{~mm}$ focus enhancement in the pineal gland.

The initial Magnetic Resonance Imaging (MRI) of the spine demonstrated two homogeneously enhancing IDEM lesions. The lesion at T2 measured $1.0 \times 1.3 \times 2.6 \mathrm{~cm}$ (Figures 1-A \& 1-B), containing a $4 \mathrm{~mm}$ non-enhancing area causing severe spinal canal stenosis with cord compression. The lesion at T12 measured $1.4 \times 0.8 \times 1.6 \mathrm{~cm}$ and caused moderate spinal canal narrowing (Figures 1-C \& 1-D). There was no obvious dural tail, expansion of neural foramina, or spinal remodeling. The follow-up brain MRI revealed a 9-mm focus of enhancement in the pineal gland, and a 4-mm focus of enhancement along the right aspect of the pituitary stalk insertion in the superior sella (Figures 1-E \& 1-F). Cerebrospinal Fluid (CSF) analysis demonstrated rare atypical lymphocytes with plasmacytoid features. Laboratory studies were otherwise unremarkable.

The patient underwent intradural and extradural thoracic tumor resection with laminectomy at T1/T2/T3 for the T2 tumor; T12/L1 for the T12 tumor via two separate incisions with the posterior spinal fusion of C7-T4, and T10-L2. The tumor was noted to be adherent to the lateral and ventral aspects of the spinal cord. Neuromonitoring outlined no changes throughout the procedure. There were no postoperative complications.

The histopathological examination of both spinal cord masses revealed the following data: Glial Fibrillary Acidic Protein (GFAP) positive, Oligodendrocyte Transcription Factor 2 (OLIG 2) negative, hypercellularity, cytological anaplasia, brisk mitotic activity, tumor necrosis, and microvascular proliferation. Genetic testing revealed MYC$\mathrm{N}$ amplification. The final pathology of spinal cord masses was high-grade glial neoplasm, consistent with the WHO grade III anaplastic ependymoma (Figure 2). The primary lesion was at $\mathrm{T} 2$, and the lesion at $\mathrm{T} 12$ represented a drop metastasis. The lesions in the pineal gland and lateral pituitary stalk were thought to be induced by tumor seeding along the CSF pathways; therefore, they were never evaluated via surgical biopsy. 


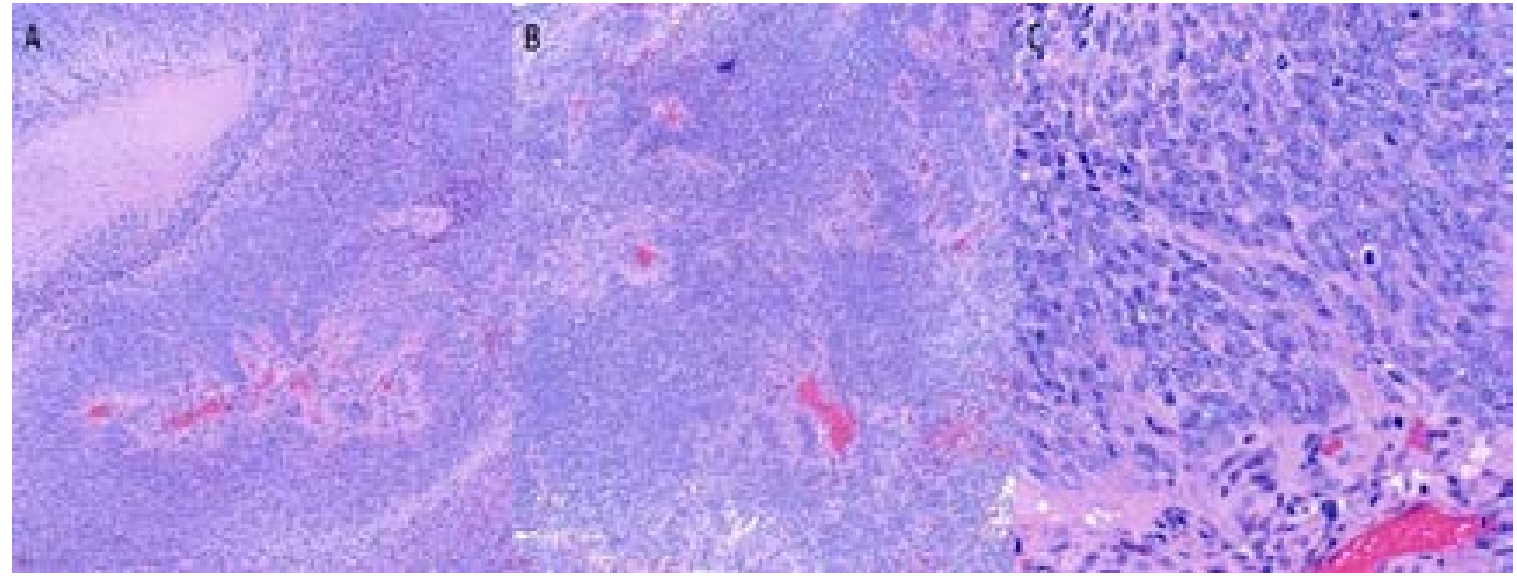

Figure 2. Low- and high-power view

A) Low-power view shows a cellular glial neoplasm with pseudo-palisading necrosis; B) Low-power view shows multiple foci of microvascular proliferation, and perivascular pseudorosette formation; C) High-power view shows marked cytological atypia, and mitoses.

The postoperative imaging of the thoracic spine demonstrated the gross total resection of the lesions without any evidence of residual enhancement on T1-weighted post-contrast MRI studies (Figure 3). Additionally, there was a resolution of spinal cord compression at the affected levels. Following two weeks of acute inpatient rehabilitation, the reported patient was discharged home with plans to receive adjuvant radiotherapy. He also regained bladder function and no longer required self-catheterization. The study patient received 36 Gy of Complete Spine Irradiation (CSI), followed by $60 \mathrm{~Gy}$

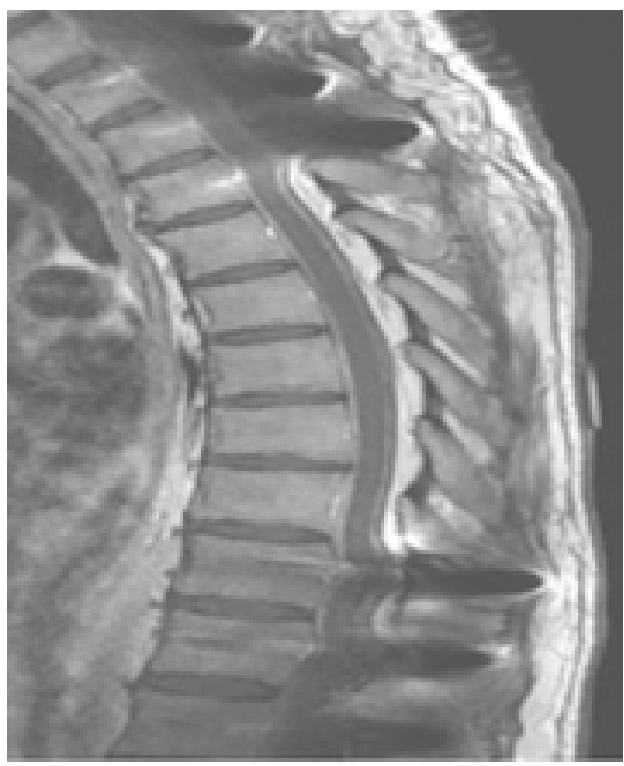

Figure 3. Postoperative sagittal T1-weighted

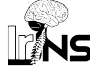

Post-contrast image of the spine demonstrating post-surgical hardware with no residual enhancement, and interval decompression of the spinal cord. of Intensity-Modulated Radiation Therapy (IMRT) to the brain, and $54 \mathrm{~Gy}$ to the spine. Imaging studies at the 10-month follow-up visit revealed no evidence of lesions neither intracranially nor in the spinal cord. His complaints included only residual patchy areas of numbness on his left thigh. The patient will undergo regular $\mathrm{MRI}$ surveillance to evaluate for disease recurrence.

\section{Discussion}

Given their rarity, much of the current knowledge about multifocal IDEM anaplastic ependymomas is derived from case reports [12-14]. Schuurmans et al. described the first multifocal IDEM anaplastic ependymoma in a 29-year-old female who presented with bilateral arm weakness and characteristic Lhermitte's sign, with tumors located in the cervical and lumbar regions of the spinal cord [12]. Following surgical resection, the patient received adjuvant radiotherapy (20 sessions of $1.8 \mathrm{~Gy}$. Two years later, an MRI revealed a tumor in the right temporal lobe; accordingly, the pathological diagnosis was consistent with anaplastic ependymoma [12]. Chakravorty et al. presented the case of a 47-year-old female with multifocal IDEM anaplastic ependymoma which initially presented with $>10$ lesions in the cervical, thoracic, and lumbosacral levels [13]. Upon diagnosis, the patient underwent surgical resection of three S2 lesions due to symptoms. One year later, she received surgery on three T5 lesions due to their progression, followed by full craniospinal irradiation with $46 \mathrm{~Gy}$. At the 48-month follow-up visit, her remaining lesions were stable, and she was neurologically intact [13]. Following the surgical resection of spinal tumors and adjuvant 
radiation, our patient was in remission at the 10-month follow-up visit. The aforementioned studies [12, 13] and this report highlighted the importance of routine radiographic evaluation; Schuurmans et al. reported intracranial metastasis two years after treatment [12]. Besides, Chakravorty et al. noted the progression of previously stable tumors one year after diagnosis [13].

It is common practice to administer adjuvant radiotherapy to patients with the WHO grade II ependymomas who undergo Subtotal Resection (STR), and those with the WHO grade III ependymomas $[11,15]$. In a series of 114 adult patients with the WHO grade II intracranial ependymomas, adjuvant radiotherapy was associated with improved survival in patients with incompletely resected tumors [15]. Nevertheless, the dosage of radiation therapy is an essential interventional component to consider. In this regard, Pica et al. demonstrated high-dose (>50.4 Gy) radiotherapy to be associated with lower rates of tumor progression in a series of 85 patients [11]. Our patient received CSI (36 Gy), followed by IMRT to the brain and spine (60 Gy and $54 \mathrm{~Gy}$ respectively). The MRI findings revealed neither intracranial nor spinal recurrence at the 10-month follow-up visit; thus, these data suggested the effectiveness of higher doses of radiation therapy in preventing recurrence, albeit with the limited follow-up length.

\section{Conclusion}

Multifocal anaplastic ependymomas are an exceedingly rare entity. To the best of our knowledge, this is the fourth report of a multifocal IDEM anaplastic ependymoma. Despite their rarity, these tumors should be considered on the differential diagnosis when evaluating a patient with spinal tumors. Careful imaging of the entire neuroaxis should be performed to investigate the presence of additional lesions at distant sites. GTR and adjuvant radiotherapy appear to be effective treatment strategies for high-grade lesions.

\section{Ethical Considerations}

\section{Compliance with ethical guidelines}

Written informed consent was obtained from the study subject, and the study was approved by the Institutional Review Board (IRB). All protected health information was secured according to the Health Insurance Portability and Accountability Act (HIPAA). The study protocol conforms to the ethical guidelines of 1975 .

\section{Funding}

This research did not receive any grant from funding agencies in the public, commercial, or non-profit sectors.

\section{Authors' contributions}

Conception and design, data collection, data interpretation, drafting the article, critically revising the article, reviewing submitted version of manuscript, approving the final version of the manuscript: Khashayar Mozaffari; Conception and design, data collection, data interpretation, drafting the article, critically revising the article, reviewing submitted version of manuscript, approving the final version of the manuscript: Michael A. Stellon; Critically revising the article, reviewing submitted version of manuscript, approving the final version of the manuscript: Eric J. Chalif; Conception and design, data interpretation, evising the article, reviewing submitted version of manuscript, approving the final version of the manuscript: Michael K. Rosner.

\section{Conflict of interest}

The authors declared no conflict of interest.

\section{References}

[1] Lee SH, Chung CK, Kim CH, Yoon SH, Hyun SJ, Kim KJ, et al. Long-term outcomes of surgical resection with or without adjuvant radiation therapy for treatment of spinal ependymoma: A retrospective multicenter study by the Korea Spinal Oncology Research Group. Neuro-oncology. 2013; 15(7):921-9. [DOI:10.1093/neuonc/not038] [PMID] [PMCID]

[2] Schellinger KA, Propp JM, Villano JL, McCarthy BJ. Descriptive epidemiology of primary spinal cord tumors. Journal of Neuro-Oncology. 2008; 87(3):173-9. [DOI:10.1007/s11060-0079507-z] [PMID]

[3] Keil VC, Schmitt AJ, Martin SC, Cadoux-Hudson TAD, Pereira EAC. Optimising treatment strategies in spinal ependymoma based on 20 years of experience at a single centre. Journal of Clinical Neuroscience. 2016; 29:52-8. [DOI:10.1016/j.jocn.2016.01.003] [PMID]

[4] Oh MC, Kim JM, Kaur G, Safaee M, Sun MZ, Singh A, et al. Prognosis by tumor location in adults with spinal ependymomas. Journal of Neurosurgery. Spine. 2013; 18(3):226-35. [DOI:10.3171/2012.12.SPINE12591] [PMID]

[5] Ostrom QT, Gittleman H, Liao P, Vecchione-Koval T, Wolinsky Y, Kruchko C, et al. CBTRUS Statistical Report: Primary brain and other central nervous system tumors diagnosed in the United States in 2010-2014. Neuro-oncology. 2017; 19(suppl_5):v1-88. [DOI:10.1093/neuonc/nox158] [PMID] [PMCID]

[6] Milano MT, Johnson MD, Sul J, Mohile NA, Korones DN Okunieff $\mathrm{P}$, et al. Primary spinal cord glioma: A surveillance, 
epidemiology, and end results database study. Journal of Neuro-oncology. 2010; 98(1):83-92. [DOI:10.1007/s11060-0090054-7] [PMID]

[7] Liu X, Sun B, Xu Q, Che X, Hu J, Gu S, et al. Outcomes in treatment for primary spinal anaplastic ependymomas: A retrospective series of 20 patients. Journal of Neurosurgery. Spine. 2013; 19(1):3-11. [DOI:10.3171/2013.3.SPINE12183] [PMID]

[8] Reni M, Gatta G, Mazza E, Vecht C. Ependymoma. Critical Reviews in Oncology/Hematology. 2007; 63(1):81-9. [DOI:10.1016/j.critrevonc.2007.03.004] [PMID]

[9] Tobin MK, Geraghty JR, Engelhard HH, Linninger AA, Mehta AI. Intramedullary spinal cord tumors: A review of current and future treatment strategies. Neurosurgical Focus. 2015; 39(2):E14. [DOI:10.3171/2015.5.FOCUS15158] [PMID]

[10] Gilbert MR, Ruda R, Soffietti R. Ependymomas in adults. Current Neurology and Neuroscience Reports. 2010; 10(3):240-7. [DOI:10.1007/s11910-010-0109-3] [PMID]

[11] Pica A, Miller R, VillÀ S, Kadish SP, Anacak Y, Abusaris $\mathrm{H}$, et al. The results of surgery, with or without radiotherapy, for primary spinal myxopapillary ependymoma: A retrospective study from the rare cancer network. International Journal of Radiation Oncology, Biology, Physics. 2009; 74(4):1114-20. [DOI:10.1016/j.jirobp.2008.09.034] [PMID]

[12] Schuurmans M, Vanneste JAL, Verstegen MJT, van Furth WR. Spinal extramedullary anaplastic ependymoma with spinal and intracranial metastases. Journal of Neuro-oncology. 2006; 79(1):57-9. [DOI:10.1007/s11060-005-9114-9] [PMID]

[13] Chakravorty A, Frydenberg E, Shein TT, Ly J, Earls P, Steel T. Multifocal intradural extramedullary anaplastic ependymoma of the spine. Journal of Spine Surgery. 2017; 3(4):727-31. [DOI:10.21037/jss.2017.11.10] [PMID] [PMCID]

[14] Ben Bouallègue F, Vauchot F, Menjot de Champfleur N, Mariano-Goulart D. Pineal anaplastic ependymoma with multifocal intradural extramedullary metastases on MRI and 18FDG-PET. Clinical Nuclear Medicine. 2018; 43(9):e319-21. [DOI:10.1097/RLU.0000000000002194] [PMID]

[15] Metellus P, Guyotat J, Chinot O, Durand A, Barrie M, Giorgi R, et al. Adult intracranial WHO grade II ependymomas: Long-term outcome and prognostic factor analysis in a series of 114 patients. Neuro-oncology. 2010; 12(9):976-84 [DOI:10.1093/neuonc/noq047] [PMID] [PMCID] 